\title{
Charles Chossat (1796-1875), physiologiste, médecin et homme politique genevois
}

\author{
Par Jean Jacques Dreifuss
}

La physiologie en tant qu'activité professionnelle n'existe à Genève ${ }^{1}$ qu'à partir de 1876, quand Moritz Schiff (1823-1896), qui était d'origine allemande et qui professait à Florence y fut appelé à Genève. Il occupera la chaire de physiologie à la Faculté de Médecine pendant vingt ans, jusqu'à sa mort en 1896.

Le présent article est consacré aux antécédents de cette physiologie à plein temps. La vie et l'œuvre de deux médecins genevois illustrent la situation précaire dans laquelle se trouvait la physiologie expérimentale, notamment durant la première moitié du $\mathrm{XIX}^{\mathrm{e}}$ siècle: Jean-Louis Prevost l'aîné (1790-1850) et Charles Chossat (1796-1875).

Moins connue que celle de son illustre compatriote et ami, l'œuvre scientifique de Chossat est aussi plus facile à cerner, parce qu'elle est plus homogène et plus limitée. Avec celle-ci, nous avons affaire à une physiologie au sens encore actuel que prendra ce terme lorsque de «médicale» elle sera devenue «expérimentale» et que l'histologie, l'embryologie, la biochimie et la pharmacologie s'en seront séparées et auront acquis leur indépendance.

Chossat n'a fait l'objet récemment, à ma connaissance, que de la seule étude - assez sommaire - que constitue la thèse de doctorat de Kurt Kilgus ${ }^{2}$, présentée à Zurich en 1967. Le texte le plus riche en informations paru à ce jour sur Chossat demeure en fait l'article, sous-titré Un médecin physiologiste, que la rédaction du Bulletin de la Société médicale de la Suisse Romande lui a consacré au lendemain de sa mort ${ }^{3}$. Nous nous basons sur cet article pour résumer brièvement quelques moments de la vie de Chossat, après quoi nous analyserons ses principaux travaux physiologiques. Nous donnons par ailleurs en annexe la liste complète de ses publications, ainsi que le texte de cinq lettres inédites ${ }^{4}$. 


\section{Formation, voyages, recherches physiologiques}

Le grand-père de Chossat, issu d'une famille française originaire du Vivarais, s'était réfugié à Genève pour des motifs religieux, puis, par la suite, fixé à Carouge. C'est là que naquit Charles, le 30 avril 1796. Celui-ci fit ses premières classes chez Pestalozzi à Yverdon, puis entra à l'Académie de Genève. En 1813, Genève était française et Chossat aurait été enrôlé dans les gardes en raison de son âge si son père ne l'avait fait inscrire comme futur étudiant en théologie à Genève et à Montauban. Tout en feignant de se préparer pour cette discipline, Chossat étudiait l'anatomie et assistait à des dissections à l'insu de son père, mais avec la complicité de Jean-François Coindet ${ }^{5}$, alors médecin-chef de l'Hôpital de Genève.

Contrairement à Prevost, qui fit trois ans de théologie avant d'entreprendre des études de médecine, Chossat, lui, se décide tout de suite pour la médecine et se rend à cette fin en 1815 à Paris. D'emblée il est un auditeur assidu de François Magendie (1783-1855), ainsi que l'atteste le texte de la lettre I qui figure en annexe au présent travail. Chossat suit aussi divers enseignements dans les sciences et s'intéresse à la marche des rayons dans les milieux de l'œil (Cuvier lui procura pour ces études d'optique un œil provenant d'un éléphant mort du Jardin des Plantes). Il assiste aux séances de la Société philomatique, y lit ses premiers travaux, y rencontre William Edwards ${ }^{6}$, élève de Magendie et auteur d'un recueil de travaux personnels dont le titre vaut tout un programme: De l'Influence des agents physiques sur la vie. Linfluence de trois hommes - Prevost, Magendie, Edwards - sera décisive pour Chossat: il se vouera à la physiologie expérimentale.

En 1818, Prevost et Chossat étudient la mort par le froid chez le chien, la thèse de doctorat que Prevost venait de soutenir à Edimbourg ayant pour sujet les bains et les affusions, mis à la mode par les travaux de James Currie (1756-1805) sur le traitement des fièvres par l'aspersion d'eau. Ce sont les seules expériences effectuées en commun par les deux Genevois, et les résultats n'en seront pas publiés. C'est Chossat seul qui continuera ces recherches à Paris.

De retour à Genève, Prevost s'attache la collaboration du jeune J.B. Dumas (1800-1884), alors simple employé à la pharmacie Le Royer et qui allait devenir l'illustre chimiste et homme d'Etat français. On sait que leurs travaux sur la biologie du développement occupent une place durable dans l'histoire des sciences. Prevost a fait l'objet de nombreuses études, mais ce 
sont surtout ses contributions sur la fécondation et l'embryologie qui ont été étudiées. Il conviendrait de s'intéresser de plus près à Prevost médecin et créateur du Dispensaire de Genéve - l'époque est celle d'une «âge d'or» de la médecine genevoise ${ }^{7}$ - ainsi qu'à ses recherches physiologiques.

En 1820, Chossat obtient le titre de docteur en médecine de Paris, avec une thèse sur l'Influence du système nerveux sur la chaleur animale. Puis il voyage en Angleterre $^{8}$, rendant visite à Londres entre autres à Benjamin Brodie, dont les travaux de 1813 sur la déperdition de chaleur chez les chiens porteurs de lésions cérébrales avaient inspiré la thèse de Chossat.

Jusqu'en 1828, année où il revient s'établir définitivement dans sa ville natale ${ }^{9}$, Chossat sera souvent en route, au titre de médecin particulier d'une comtesse autrichienne qui voyageait en Allemagne, en France et surtout en Italie, où elle avait l'habitude de séjourner en hiver. Les recherches de Chossat se ressentent de ce nomadisme. Tandis que la thèse de 1820 est un travail classique de vivisection, entrepris sur des chiens, ceux qui suivront seront par nécessité les œuvres d'un expérimentateur itinérant, dont les instruments doivent être légers et portatifs; une physiologie démunie, où la pauvreté des moyens techniques sera compensée par le nombre des mesures et la rigueur du raisonnement.

Chossat consacra à l'auto-expérimentation la période qui va de 1821 à 1824. Il en résultera un épais Mémoire sur l'analyse des fonctions urinaires, qui parut dans le Journal de Physiologie expérimentale et pathologique et qui vaudra à Chossat d'obtenir le prix Montyon de physiologie expérimentale pour 1825. Ce prix était attribué annuellement depuis 1820 par l'Académie des Sciences. A un moment où seule l'anatomo-physiologie humaine avait droit de cité à la Faculté de médecine de Paris sous l'égide de Chaussier (1746-1828), Richerand (1779-1840) et Bérard (1797-1858), il couronnait systématiquement des savants qui préfiguraient la nouvelle physiologie expérimentale de l'école de Magendie ou des expérimentateurs comme Flourens (1794-1867) ${ }^{10}$.

A partir de 1825 environ, Chossat entreprit ses Recherches expérimentales sur l'inanition, qu'il enverra à l'Académie des Sciences de Paris en 1838. A nouveau, le Prix Montyon de physiologie expérimentale - celui de 1841 viendra récompenser ce travail. Long de plus de 200 pages in $-4^{\circ}, c^{\prime}$ est à la fois le principal ouvrage de Chossat et son dernier, à l'exception de deux courtes notes qui ont rapport au même sujet et qui parurent en 1842 et 1843 dans les Comptes-rendus de l'Académie des Sciences. La date d'envoi des Recherches à l'Académie des Sciences est trompeuse, Chossat en ayant déjà certainement 
terminé le travail expérimental au début de la décennie, probablement vers 1833 quand il se maria ${ }^{11}$. Deux filles naîtront de son union avec Sara Du Roveray (1810-1888), Pauline en 1834 et Mathilde en 1844. Suivra un fils, Théodore (1845-1934), qui deviendra médecin comme son père.

Nous n'analyserons ici que les deux travaux qui ont été distingués par l'attribution du Prix Montyon. Nous passerons donc sous silence les travaux sur l'optique visuelle, que Chossat entreprit en tant qu'étudiant. Quant à sa thèse, l'Influence du système nerveux sur la chaleur animale, c'est le résultat d'une recherche rigoureuse, mais assez sommaire et dont l'intreprétation n'a pas résisté à l'usure du temps. Ils s'agissait d'expériences où une trentaine de chiens subirent une transection expérimentale de leur système nerveux central ou périphérique - et où Chossat notait la marche horaire du refroidissement corporel, lequel aboutissait, en une dizaine ou une quinzaine d'heures, à la mort de l'animal ${ }^{12}$.

\section{L'analyse des fonctions urinaires}

C'est un travail autrement plus original et plus riche, long de plus de cent pages in- $8^{\circ}$. Il fut entrepris dans les conditions difficiles qu'imposait à Chossat sa condition de médecin d'une malade itinérante ${ }^{13}$. Comme instruments de mesure, il utilisa une balance pour peser la nourriture qu'il absorbait, un vase gradué pour mesurer le volume de ses urines et un aréomètre pour en déterminer le poids spécifique.

Chossat s'était donné pour but de distinguer la part dans l'excrétion urinaire qui dépend de facteurs endogènes de celle qui est surbordonnée à des facteurs exogènes. Outre la température cutanée - souvenir de ses expériences avec Prevost sur le bain froid - un facteur «externe» a surtout retenu son attention: la nutrition. Dans une première série d'expériences il étudie l'effet de la quantité des aliments ingérés, ainsi que de leur nature soit végétale soit animale - sur la quantité des solutés urinaires. A côté de ces relations quantitatives, il s'intéresse aussi aux correspondances dans le temps et, à cette fin, compare son excrétion urinaire par périodes de huit heures selon qu'il ingérait un ou trois repas quotidiens, voire un seul repas tous les deux jours.

Dans une seconde partie de ce travail, il s'attache aux facteurs «internes» qui peuvent influer sur l'excrétion urinaire. Pour ce faire, il choisit un régime alimentaire composé de trois repas égaux et pris à intervalles réguliers de 
huit heures, de façon à éliminer dans toute la mesure du possible l'effet de la prise d'aliments. Cette approche lui permet de montrer l'existence d'une variation diurne - nous dirions aujourd'hui d'un rythme circadien - de l'excrétion urinaire: diminution vers la fin du jour, augmentation pendant le sommeil et la première partie de la journée. Il imagine également une autre expérience pour préciser l'influence de facteurs «internes» sur l'excrétion urinaire. Pour ce faire il se privait de sommeil pendant certaines nuits et comparait sa production d'urine avec celle de jours où il s'accordait du sommeil. Au vu des observations recueillies, Chossat postule que le système nerveux central influe sur les mécanismes d'excrétion urinaire (comme il pensait avoir montré préalablement qu'il influait sur la chaleur animale).

\section{Recherches expérimentales sur l'inanition}

Dans son travail sur les fonctions urinaires, Chossat avait noté qu'il lui était plus facile de mettre en évidence l'influence diurne - c'est-à-dire la variation de l'excrétion urinaire selon l'alternance jour/nuit - s'il diminuait pendant quelques jours sa prise d'aliments. Pour se rendre complètement indépendant du facteur alimentaire, il lui aurait fallu jeûner pendant de longues périodes.

Les Recherches expérimentales sur l'inanition s'inscrivent dans la droite ligne de ces réflexions. C'est un travail d'observation patiente, où Chossat note quotidiennement, des semaines durant, l'évolution du poids et de la température corporelle chez des animaux qui sont nourris normalement ou qui sont - soit partiellement soit entièrement - privés de nourriture. C'est un travail qui s'étend sur plusieurs années et Chossat utilisera pour le réaliser au moins une quarantaine de pigeons, une trentaine de tourterelles, deux poules, une corneille, cinq cobayes, cinq lapins, douze grenouilles, trois reinettes, une tortue, trois anguilles, trois couleuvres et six lézards!

Plutôt que de suivre l'ordre de l'exposé de Chossat, voyons tout d'abord la seconde partie, qui touche aux effets de l'inanitiation ${ }^{14}$ sur la chaleur animale. Chossat s'y inscrit en faux contre la notion unanimement admise à l'époque, selon laquelle les animaux à sang chaud possèdent une température corporelle toujours égale. Les observations qu'il a effectuées en 1831 sur une vingtaine de pigeons l'avaient en effet amené à découvrir l'existence d'une oscillation circadienne de la température corporelle ${ }^{15}$ : indépendam- 
ment des modifications de la température ambiante, la température corporelle prise à midi dépassait celle prise à minuit de $0,7^{\circ} \mathrm{C}$ en moyenne. Chossat se demande ensuite si ce rythme circadien de la température corporelle est modifié par la privation de nourriture. Dix des vingt pigeons qui ont servi à l'expérience précédente seront donc privés de nourriture. Leur poids diminue progressivement, ainsi que leur température corporelle: Chossat observe que cette baisse ne touche que peu la température prise à midi, mais fortement la température nocturne.

Voici le résumé que Chossat fait de ces expériences: L'on voit (...) la chaleur animale osciller régulièrement chaque jour, s'abaissant le soir de quelques degrés, et remontant le matin à l'état où elle était la veille, et osciller avec une amplitude graduellement croissante à mesure que l'abstinence se prolonge et que la faiblesse fait des progrès. Ce fait, d'autant plus curieux qu'il n'est que le développement d'un phénomène presqu'inaperçu à l'état normal, prouve avec évidence que les combinaisons d'où résulte le dégagement de la chaleur animale se font essentiellement sous l'influence nerveuse, (...) plus particulièrement sous l'influence du cerveau, puisque cet organe est le siège spécial de la remittance d'action d'où résulte l'état de veille et celui de sommeil ${ }^{16}$.

Dans la première partie des Recherches, Chossat avait montré que des mammifères, des oiseaux, des batraciens et des reptiles mouraient lorsque, du fait de la privation de nourriture et d'eau, ils avaient perdu environ quatre dixièmes de leur poids corporel initial. Chossat, se demandant comment les divers organes et tissus étaient affectés, entreprit alors l'expérience suivante: Je me suis successivement procuré dix paires de pigeons, choisis de façon que les deux animaux de chaque paire fussent autant que possible de même âge et de même poids. Dans chaque paire, l'un des animaux a été asphyxié par strangulation; l'autre a été inanitié par privation des aliments et des boissons. De cette manière, nous avons eu à comparer dix autopsies d'animaux morts dans l'état normal de la nutrition (asphyxie), avec dix autopsies (...) d'animaux semblables morts par inanitiation ${ }^{17}$. A l'autopsie, les organes et tissus étaient pesés aussi rapidement que possible, de manière à éviter au maximum toute dessication ${ }^{18}$.

Les résultats obtenus ne manquent pas d'intérêt, qu'ils soient exprimés en valeur absolue ou en valeur proportionnelle. Les pigeons, qui pesaient initialement dans les $380 \mathrm{~g}$ en perdaient plus de 140 pendant la période de privation.

Les pertes se répartissaient comme suit: 


$\begin{array}{lr}\text { muscles locomoteurs } & 66,3 \mathrm{~g} \\ \text { graisse } & 38,5 \mathrm{~g} \\ \text { sang } & 7,9 \mathrm{~g} \\ \text { système glandulaire abdominal } & 7,5 \mathrm{~g} \\ \text { appareil musculaire de la digestion } & 6,4 \mathrm{~g} \\ \text { peau } & 5,6 \mathrm{~g} \\ \text { os } & 5,3 \mathrm{~g} \\ \text { autres } & 4,6 \mathrm{~g} \\ \text { Total } & 142,1 \mathrm{~g}\end{array}$

Chossat note que si la perte proportionnelle est en moyenne de $40 \%$, il y a des tissus dont le poids diminue plus considérablement $(93,3 \%$ de la graisse, $61,1 \%$ du pancréas et $52 \%$ du foie ont disparu); et, à l'opposé, d'autres que l'inanition n'affecte que peu (les pertes par rapport au poids initial s'élèvent à $1,9 \%$ pour le système nerveux, $10 \%$ pour les yeux, $16,7 \%$ pour le squelette et $31,9 \%$ pour le rein, par exemple).

Il nous faut admirer la simplicité des moyens, la rigueur du dessein expérimental, la persévérance d'une recherche solitaire ${ }^{19}$, le grand nombre des mesures et la finesse de l'analyse. On comprend que ce travail, dont je n'ai résumé qu'une petite fraction, ait retenu l'attention des membres de l'Académie des sciences de Paris. Les médecins, plus pragmatiques, y trouvaient également leur compte s'ils retenaient la conclusion suivante: Puisque l'alimentation insuffisante a, sauf pour la durée, identiquement les mêmes effets d'inanitiation que l'abstinence absolue, il est clair que, dès que l'alimentation devient, je ne dirai pas suspendue, mais seulement diminuée, la question d'inanitiation se soulève, et que l'inanition complète n'est plus qu'une affaire de temps. L'inanitiation (...) est la cause de mort qui marche de front et en silence avec toute maladie dans laquelle l'alimentation n'est pas à l'état normal. Elle arrive à son terme naturel, quelquefois plus tôt et quelquefois plus tard que la maladie qu'elle accompagne sourdement, et peut devenir ainsi maladie principale, là où elle n'avait été d'abord qu'épiphénomène. On la reconnâ̂tra (...) au degré de destruction des chairs ${ }^{20}$. Rappelons que Chossat vivait à une époque qui pratiquait volontiers les saignées et la mise au régime des malades. Aussi a-t-il raison de conclure: Je soulève ici des questions du plus haut intérêt dans l'état actuel de la médecine, mais la grande étendue de ce Mémoire ne me permet pas de les examiner. Je compte cependant y revenir un jour $^{20}$.

Il n'y reviendra pas, malheureusement. A partir de 1840 environ, la vie de Chossat ne concerna plus guère la physiologie. Dans la Genève du 
XIX $^{\mathrm{e}}$ siècle, on ne pouvait pas faire de la physiologie quand on n'avait pas de fortune et une famille à nourrir. Prevost, qui demeura sa vie durant célibataire et qui était d'une famille plus aisée, cessa aussi de publier une décennie durant, mais revint par la suite au microscope; à partir de $1837 \mathrm{il} \mathrm{se}$ retira de la pratique médicale et s'installa dans une propriété familiale située hors de la ville. Prevost mourut, bien avant Chossat, en 1850. Il ne vécut donc pas assez longtemps pour connaître toutes les transformations que subit Genève à la suite de la Révolution de 1846, avec l'arrivée au pouvoir des Radicaux et de leur homme fort, James Fazy.

\section{Chossat dans la Genève radicale}

Elu comme député radical au Grand Conseil instauré par la Constitution de 1842, Chossat devint membre du Conseil d'instruction publique dont le mandat était d'organiser et de superviser toutes les activités d'enseignement dans le canton. Il fut également rapporteur d'un triumvirat dont les travaux aboutirent à des propositions sur l'organisation d'un enseignement de niveau secondaire pour les élèves se destinant aux métiers du commerce et de l'industrie. Mais, l'année même de la parution du rapport, éclata la Révolution qui - en octobre 1846 - devait voir triompher les Radicaux.

Le Conseil d'instruction publique fut dissous et ses attributions confiées à un conseiller d'Etat, chargé du Département de l'instruction publique. Le premier titulaire en fut A.-L.Pons qui, au début de 1847, nomma des délégués généraux pour servir d'intermédiaires entre ses services et les différents établissements d'enseignement. Chossat fut délégué auprès de l'Académie, Pons estimant que celui-ci s'entendrait avec les professeurs restés en place et avec le nouveau recteur, F.-J.Pictet de la Rive (1809-1872). La mission de Chossat fut précisée dans une lettre datée du 24 janvier 1847:

«A M. le Docteur Chossat, délégué général près l'Académie. Comme vous avez eu l'extrême obligeance d'accepter les fonctions de délégué général du Département de l'Instruction publique près l'Académie, j'ai l'honneur de vous transmettre par écrit copie des attributions générales qui vous sont confiées en cette qualité: 1 . Assister aussi souvent que vous le jugerez convenable à la séance ordinaire où $M$. le Recteur fait au Département son rapport sur la marche et l'état de l'enseignement (une carte de convocation vous avertira à cet effet du jour et de l'heure où cette séance a lieu). 2. Veiller 
et au besoin rappeler à l'exécution des lois, règlements et arrêtés relatifs à l'Académie, dans tout ce qu'ils ont de conforme à la constitution, et surveiller l'emploi des dons, legs et sommes affectées à cet établissement. 3. Prendre à titre de renseignement, et selon le mode que vous jugerez le plus convenable, le préavis du Corps académique ou des Facultés sur tout objet au sujet duquel le Département désirerait être éclairé. 4. Assister toutes les fois que vous le trouverez bon aux cours académiques. 5 . Adresser directement au Département toutes les observations ou propositions qui vous paraîtront utiles à l'Etablissement. 6. En un mot représenter le Département de l'Instruction publique et lui servir d'intermédiaire auprès de l'Académie, toutes les fois que cela sera nécessaire. - Je me plais à croire qu'avec M. le Recteur, la responsabilité du Département et la délégation que vous avez bien voulu accepter ne donneront lieu qu'à des rapports agréables et favorables en tous points au bien des études et de l'enseignement. Agréez, etc. ... ${ }^{21}$.

Pons fit également appel à Chossat lorsqu'il créa, en juin 1847, une commission de cinq membres chargée d'élaborer, pour le projet de la Loi sur l'instruction publique, la partie qui touchait à l'organisation de l'Académie. Chossat devait probablement cette nouvelle nomination non seulement au fait qu'il s'acquittait à satisfaction de la mission d'intermédiaire entre le Pouvoir et l'Académie qui lui était confiée mais aussi parce qu'il pouvait s'exprimer au nom des docteurs en médecine, auxquels le gouvernement envisagea un temps de faire une place dans l'Académie.

La nouvelle loi sur l'instruction publique fut adoptée par le Grand Conseil en octobre 1848. Elle prévoyait la création à la Faculté des sciences et des lettres d'une chaire d'anatomie humaine et de physiologie. Ce ne furent ni Prevost ni Chossat qui furent nommés, mais un chirurgien septuagénaire, François-Isaac Mayor père (1779-1854), qui avait de 1835 à 1837 enseigné la médecine légale à la Faculté de droit et était lié à James Fazy par l'amitié et la politique. S'estimant trop âgé, Mayor refusa sa nomination ${ }^{22}$. La chaire revint à son fils et homonyme, François-Isaac Mayor (1818-1899). Médecin et politicien apprécié, représentant type de l'anatomo-physiologie médicale, Mayor n'avait pas l'étoffe d'un physiologiste original et n'a pas contribué beaucoup à l'avancement de la physiologie expérimentale à l'Académie ${ }^{23}$. Il joua par contre un rôle dans le genèse d'une institution importante issue de la Révolution radicale: l'Hôpital cantonal. De 1849 à 1856, soit pendant toute la période qui va de la décision prise par le Grand Conseil à l'inauguration du bâtiment qui fut édifié à La Cluse, il siégea dans la commission administra- 
tive de l'hôpital. Puis de 1856 à 1860 , il en fut le chirurgien en chef. Mayor se fit également entendre dans les débats qui devaient aboutir à la création de l'Hospice Général en 1869.

Il nous faut revenir à Chossat, encore que nous ne disposions que de peu de renseignements sur ses dernières années. En 1858, il fit pour les Archives de la Bibliothèque universelle l'analyse d'un ouvrage de recherches chimiques et physiologiques entreprises sur des vertébrés d'Amérique. En 1863-1864, il présida la Société de physique et d'histoire naturelle; il y était entré comme membre en 1830, l'année où il avait démissionné de la Société médicale de Genève, deux ans seulement après y avoir été admis. J.-L. Prevost aussi n'en fut qu'un membre éphémère: admis en 1825, président en 1826, il en démissionna en 1832. Il serait intéressant de connaître si des raisons plus profondes que celles qui figurent dans la lettre de démission de Chossat (Lettre V) expliquent pourquoi nos deux médecins physiologistes ne s'y sont pas senti à l'aise. Un ultime texte de 1872 et signé par Chossat et Jean Charles Coindet ${ }^{24}$ concerne la marche de La Métairie, une clinique psychiatrique privée fondée en 1858 et qui se trouve située près de Nyon.

Nous possédons quelques témoignages sur le médecin et le lecteur assidu que fut Chossat au soir de sa vie. Nous citerons ci-dessous quelques lignes du Dr Alexandre Stroehlin (1813-1889):

«Il avait adopté tous les moyens d'investigation récents, sans toutefois y mettre d'enthousiasme, car sa longue expérience lui avait appris à se méfier de ces moyens qui doivent révolutionner la science; il en avait tant vu échouer, qu'il était devenu un peu sceptique et n'acceptait les soi-disant découvertes que sous bénéfice d'inventaire. C'était un diagnosticien très habile, quoiqu'il ne marchât pas ses poches pleines de balances et de thermomètres ou d'autres instruments, et après avoir bien pris connaissance des symptômes de la maladie, il maniait avec beaucoup de tact et de jugement les agents médicamenteux au moyen desquels il combattait le mal.» Et Stroehlin poursuit: «Chossat n'a jamais été intéressé, c'est une des raisons pour lesquelles il n'avait rien fait pour étendre sa clientèle. Il avait peu de besoins. Sauf pour ses livres il faisait peu de dépenses, et sa besogne faite il n'aimait rien tant que d'être au milieu de sa famille et de voir quelques amis de choix.» ${ }^{25}$

Un texte du professeur L. Revilliod ${ }^{26}$ suggère que ni la passion de Chossat pour la connaissance, ni son goût pour la lecture ne se sont éteintes de son vivant: «Chossat, toujours dévoré d'une soif inextinguible d'instruction, passait chaque jour, autant que ses forces le lui ont permis, de sa bibliothèque 


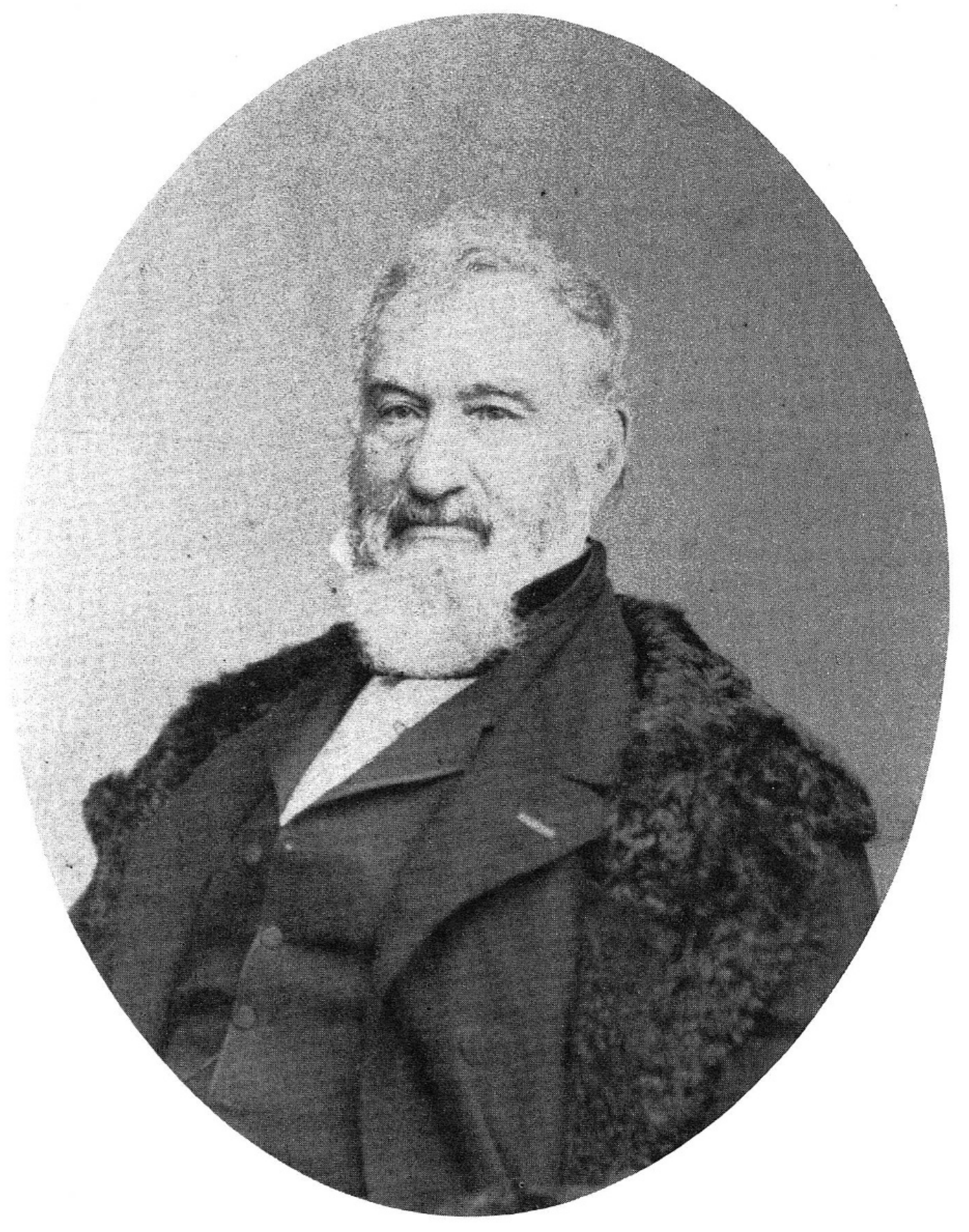

Fig. 1 Charles Chossat septuagénaire

(Photo Temporel) 
à la Société de lecture, où les habitués du matin pouvaient le voir parcourant sans relâche les revues scientifiques, littéraires et politiques, se tenant au courant de tout comme s'il avait encore une longue carrière à parcourir.» Revilliod rappelait par ailleurs «cette physionomie caractéristique taillée à grands traits, ce front sillonné de rides, cette figure expressive qui, sous une apparence sévère, soudain s'illuminait sous l'influence d'une causerie dans laquelle se révélait une vaste érudition». ${ }^{27}$

De la même période tardive date une photo de Chossat (Fig. 1) qui nous montre un homme porteur d'une barbe blanche et au revers du veston duquel on aperçoit un ruban; il reçut en effet en 1865 la Croix de la Légion d'honneur. Il était apprécié par les savants parisiens qui l'avaient nommé membre correspondant de l'Académie de Médecine en 1846. A la fin de sa vie, ses travaux scientifiques étaient encore cités en France. Dans les Leçons sur la chaleur animale, professées en 1871-1872 au Collège de France, Claude Bernard (1813-1878) cite la thèse de doctorat de Chossat dans l'historique qu'il fait de son sujet ${ }^{28}$. Chossat est également mentionné dans le Dictionnaire de Physiologie de Ch. Richet (1850-1935), dont les divers volumes parurent au tournant du siècle. Les auteurs de l'article Chaleur, faisant allusion à la température corporelle plus élevée des oiseaux par rapport aux mammifères, rappellent comme un fait solidement établi que chez les pigeons, 600 observations de Chossat donnent une moyenne de 41,9 degrés: 42,22 à midi, 41,48 à minuit ${ }^{29}$.

\section{De l'Académie à l'Université}

Chossat mourut le 7 mars 1875, âgé de 79 ans. Deux mois auparavant, Mayor fils avait démissionné de son poste de professeur à l'Académie. En septembre 1873 au Grand Conseil, il s'était rangé parmi les adversaires de la création de la Faculté de médecine, parce qu'il la trouvait trop onéreuse pour les finances publiques!

Durant l'hiver 1874-75, un cours libre sur la physiologie du sang fut annoncé par J.-L. Prevost le cadet (1838-1927), qui bénéficiait d'une solide formation aussi bien neurologique que physiologique acquise à Paris, notamment auprès de J.M.Charcot (1825-1893) et de A.Vulpian (1826-1887). Visait-il l'obtention de la chaire de physiologie à pourvoir à la Faculté de médecine? Le fait est que Prevost était déchiré entre la physiolo- 
gie et la médecine, entre le laboratoire et la consultation. Dans une lettre de 1867 , il avait confié à ses parents, à propos de son travail de doctorat: $O n$ m'accuserait si je venais à faire de la clientèle d'avoir fait une thèse de physiologie pure. Médecin en chef à l'Hôpital cantonal depuis 1874, Prevost écrit en 1876 qu'il ne saurait accepter une position qui me sortirait de la profession médicale ${ }^{30}$. Carl Vogt (1817-1895), recteur de l'Académie devenue l'Université par la création de la Faculté de Médecine, et Antoine Carteret, conseiller d'Etat chargé du Département de l'Instruction publique, préféraient d'ailleurs faire appel à une personnalité à la réputation mieux établie et venue de l'extérieur. Après une tentative infructueuse auprès de Charles-Edouard Brown-Séquard (1818-1894), Vogt se tourna vers Moritz Schiff. Avec la venue de ce dernier à Genève - et avec l'inauguration de la Faculté de Médecine en octobre 1876 - commence un nouveau chapitre de l'histoire de la physiologie genevoise ou, en d'autres termes, se termine sa préhistoire.

Quelle est la place de Chossat parmi les personnes mentionnées dans le présent travail: Prevost l'ainé, François-Isaac Mayor fils, Schiff, Prevost le cadet? Dans son étude sur les hommes de science genevois nés au XVIII siècle et dans la première moitié du XIX ${ }^{\mathrm{e}}$ siècle, Mme Montandon ${ }^{31}$ place les deux Prevost dans la catégorie des quarante savants d'importance majeure qui sont la fierté des Genevois. Chossat y est signalé, mais il figure parmi la centaine de savants de moindre importance. Les Mayor, eux, n'apparaissent pas dans ce recensement, non plus que Schiff, mais celui-ci était né à Francfort-sur-le-Main. Mon classement personnel n'est pas fondamentalement différent. J.-L. Prevost l'aîné figure en tête et Mayor en fin de liste. Je ne suis pas convaincu que $J$.-L. Prevost le cadet fut un meilleur savant que Chossat, ni son influence sur la physiologie plus durable. Certes il sut mieux se faire une place (sinon un prénom!) dans le monde savant genevois et international ${ }^{32}$ que Chossat un demi-siècle plus tôt. Mais à la fin du XIX siècle la physiologie expérimentale avait acquis ses lettres de noblesse. 


\section{Annexe 1: Publications de Ch. Chossat}

1 Mémoire sur le pouvoir réfringent des milieux de l'œil. Bulletin de la Société philomatique de Paris, 1817. Extrait dans Annales de Chimie et de Physique 8, 1818, p. 217-222.

2 Mémoire sur la courbure des milieux réfringents de l'œil chez le bœuf. Annales de Chimie et de Physique 10, 1819, p. 337-368. Extrait dans Bibliothèque universelle 9, 1818, p.26-31.

3 Mémoire sur l'influence du système nerveux sur la chaleur animale. Paris, Didot Jeune, $1820,49 \mathrm{p}$. [présenté à l'Académie des Sciences de Paris le 15 mai 1820 et thèse de doctorat le 13 juin 1820]. Extrait dans Annales de Chimie et de Physique 15, 1820, p.27-49; et, en anglais, dans Annals of Philosophy, New Series 2, 1821, p. 37-43.

4 Mémoire sur l'analyse des fonctions urinaires [Prix de Physiologie expérimentale pour 1825]. Journal de Physiologie expérimentale et pathologique 5, 1825, p.65-221.

5 Recherches expérimentales sur l'inanition [présenté à l'Académie des Sciences de Paris le 25 décembre 1838, Prix de physiologie expérimentale de Montyon pour 1841]. Mémoires présentés par divers savants à l'Académie Royale des Sciences de l'Institut de France (Sciences mathématiques et physiques) 8, 1843, p.438-640. Extraits dans Annales des Sciences naturelles (Zoologie), Seconde série, 20, p.54-81, 182-214 et 293-326.

6 Note sur le système osseux. Comptes rendus hebdomadaires des Séances de l'Académie des Sciences de Paris, 14, 1842, p.451-454. Extrait dans Annales des Sciences naturelles (Zoologie), Seconde série, 17, 1842, p. 206-209.

7 Recherches expérimentales sur les effets du régime du sucre. Comptes rendus hebdomadaires de l'Académie des Sciences de Paris, 17, 1843, p. 805-808.

8 Rapport au Conseil d'Instruction publique sur l'enseignement à fournir dans le Canton de Genève aux classes industrielles et commerçantes de la population et sur les établissements publics destinés à y pourvoir. Genève 1846, 164 p., 1 tableau.

9 Rapport sur les travaux de la Société de Physique et d'Histoire naturelle de Genève, depuis juillet 1863 à juin 1864. Mémoires de la Sociêté de Physique et d'Histoire naturelle 17, 1864, p. 501-524.

10 Rapport présenté aux actionnaires de la Société anonyme La Métairie, sur les circonstances qui ont nécessité leur convocation en Assemblée générale extraordinaire, le 14 décembre 1872. Genève, Imprimerie Carey, 1872, 48 p. (avec J. Ch. Coindet).

\section{Annexe 2: Cinq lettres de Ch. Chossat}

I. Lettre au Professeur Pierre Prevost ${ }^{33}$ à Genève. Paris, le $1^{\text {er }}$ décembre 1815. Monsieur, En prenant la liberté de vous adresser une lettre et de vous distraire des occupations sérieuses dans lesquelles vous êtes habituellement plongé, je n'ai compté que sur la bonté obligeante avec laquelle vous accueillez tous ceux qui ont eu le bonheur d'étudier sous votre direction. Cette idée seule m'a enhardi à oser vous demander votre avis sur quelques opinions nouvelles de Monsieur Magendie, Professeur de Physiologie à la Faculté de Médecine de Paris et dont je suis le cours. Ces opinions sont relatives à quelques points d'optique, et me paraissent expliquer d'une manière tout à fait ingénieuse certains points de la Vision.

1. On considère ordinairement le cristallin comme une simple lentille, mais à tort car la substance d'une lentille est partout de la même densité, au lieu que celle du cristallin augmente 
à mesure que l'on approche de son centre. Sa substance étant partout homogène, sa puissance réfringente doit être proportionnelle à sa densité. Donc la faculté réfringente du cristallin est d'autant plus grande que l'on approche davantage du centre de ce corps. Or le principe de déviation établit que toute lentille occasionne aberration de sphéricité, les rayons étant d'autant plus déviés que leur point d'incidence est plus éloigné du centre de la lentille. Mais la puissance réfringente du cristallin étant d'autant plus grande que l'on s'approche davantage du centre de ce corps, il suit de là que par le principe de déviation les rayons qui devraient être le plus déviés tomberont sur des points moins réfringents du cristallin et que ceux qui devraient l'être le moins, tomberont sur des points plus réfringents: en sorte que il y aura compensation, que tous les points se réuniront en un même foyer et que l'aberration de sphéricité sera détruite. - Telle est, selon Magendie, la cause de la différence de densité du cristallin.

2. Les larmes qui sont continuellement sécrétées par la glande lacrymale se répandent sur la cornée et $\mathrm{y}$ forment une couche très mince. Toutes les surfaces qui transmettent de la lumière en réfléchissent une portion. Or la lumière peut pénétrer au fond de l'œil ayant plusieurs milieux diaphanes à traverser, il y aura des rayons lumineux réfléchis à la surface de chacun des milieux que la lumière traverse, savoir, 1 . à la surface de la couche des larmes, 2 . à celle de la cornée, 3 . à celle de l'humeur aqueuse, 4 . à celle du cristallin, 5 . à celle de l'humeur vitrée, 6 . à celle de la rétine elle-même puisqu'elle est transparente. Ce sont ces différentes portions de lumière réfléchie qui forment le brillant des yeux. Mais la totalité de la lumière réfléchie par le cristallin, l'humeur vitrée et la rétine ne peut pas ressortir de l'œil par la prunelle. Une portion de cette lumière doit tomber sur l'iris et sur la choroïde. Si ces membranes n'étaient pas de couleur noire (c.à.d. d'un tissu tel qu'elles absorbent tous les rayons lumineux) elles réfléchiraient quelques-uns de ces rayons déjà réfléchis sur elle. Ces rayons troubleraient l'image qui doit aller se peindre sur la rétine et produiraient confusion dans la vision. C'est donc là la fin pour laquelle l'iris et la choroïde ont été enduits du vernis noir que nous lui connaissons. Les idées que je viens de vous exposer jusqu'ici sont toutes de Monsieur Magendie, mais puisqu'il me reste encore quelqu'espace, $j$ 'en profiterai pour vous demander votre avis sur deux faits que vous nous avez exposé dans vos leçons publiques, et que je crois pouvoir expliquer d'une manière un peu différente de la vôtre:

1. Lorsqu'on regarde un objet avec une lentille duplo-convexe, cet objet parâ̂t plus grand quand l'on éloigne l'æil du verre que quand on l'en approche. Il y a ici une illusion, comme vous nous l'avez fort bien fait remarquer. Lorsqu'on regarde avec l'œil rapproché du verre un certain objet comme de l'écriture, l'on voit dans le champ du microscope outre la lettre que l'on regarde un certain nombre d'autres lettres voisines. Lorsque l'on éloigne l'œil du verre toutes les autres lettres sortent du champ du microscope et il n'y reste que celle que l'on considérait particulièrement. L'œil ne remarque pas que l'étendue du champ du microscope a diminué et comme il le voit rempli par la lettre en question, il se fait illusion et juge que c'est la lettre elle-même et non le champ qui a diminué. Telle me paraît être l'explication de cette illusion d'optique. Quand on répète l'expérience très attentivement en connaissant d'ailleurs la cause de l'illusion on parvient à affaiblir beaucoup l'illusion elle-même et à trouver que l'objet ne grossit réellement pas, mais qu'il ne fait qu'occuper un plus grand espace sur le verre.

2. Pourquoi le bruit des rivières est-il plus sensible de nuit que de jour? De jour nous sommes mis en communication avec les objets éloignés au moyen de deux sens principalement, la vue et l'ouïe: comme les sensations de la vue sont beaucoup plus fortes, et peut-être dans le plus 
grand nombre des cas plus attrayantes pour l'âme que les sensations de l'ouie, l'âme est distraite pendant le jour par les sensations de la vue; celle de l'ouie ne font qu'une faible impression sur elle. Dans la nuit, c'est tout différent: l'âme ne peut plus employer que le sens de l'ouïe: il devient le supplément de la vue: ce n'est que la nuit qu'elle est mise en communication avec les objets éloignés. Aussi l'oreille redouble d'activité, et l'âme devient plus attentive aux impressions qui lui arrivent par cet organe. Aussi ces sensations doiventelles lui paraître beaucoup plus fortes que pendant le jour. Telle est l'explication que je crois pouvoir donner au fait susmentionné.

Je terminerai ici cette lettre qui n'est déjà que beaucoup trop longue sans doute. Je vous prie, Monsieur, d'excuser mon indiscrétion en faveur du motif qui l'a causée, le désir de jouir de vos précieux conseils. Si vous daignez m'honorer d'une réponse mon adresse est Rue des Maçons (près la Sorbonne) No 11 à Paris. En attendant cette faveur, j'ai l'honneur d'être Votre très humble serviteur. Charles Chossat.»

II. Lettre aux membres de la Société des Amateurs des Sciences naturelles ${ }^{34}$ à Genève. «Paris, le 26 mai 1817. Très chers Collègues, Je suis extrêmement charmé des relations qui existent entre notre société et celle qui s'est formée plus récemment et dont vous nous parlez dans la lettre que Monsieur le Président nous a fait l'honneur de nous adresser: ce seront autant de foyers dont il faudra chercher à faire jaillir la lumière, à l'envi les uns des autres, car malgré les meilleures dispositions du monde, je crois qu'il est toujours bon d'avoir un stimulus particulier, qui sans cesse nous aiguillonne et ne nous donne pas un moment de relâche: il arrivera un jour où ce sera à notre génération à soutenir chez l'étranger la réputation scientifique de notre patrie, réputation établie d'une manière si brillante par les de Saussure, les Deluc, les Hubert et tant d'autres noms appuis de la Science: et pour ne pas paraître alors trop inférieurs à eux, de quelle provision de courage, très chers collègues, ne devons-nous pas être munis. Vous m'avez fait l'honneur de communiquer à cette autre Société le mémoire que je vous ai envoyé sur l'ajustement; et vous de dites qu'ayant voulu répéter les expériences, ils n'ont pas trouvé exacts les nombres que j'ai indiqué: je commencerai par vous rappeler ce que je dis dans ce mémoire, que par certaines considérations particulières j'avais été forcé de vous l'envoyer avant l'heure où je comptais le faire; que de plus je n'avais pas à ma disposition les instruments nécessaires à des expériences très exactes; néanmoins j'ai procédé avec autant d'exactitude que me le permettait l'imperfection de mes moyens. En second lieu, je ne conçois pas comment ces Messieurs peuvent accuser l'exactitude de mes résultats n'ayant pas opéré avec la même lentille que moi; car n'ayant pas alors le moyen de déterminer le rayon de la lentille dont je me suis servi, je ne pus l'indiquer dans mon mémoire; je le ferais actuellement cependant si je me rappelais celle dont je me suis servi dans mes expériences. Enfin, en admettant même que j'ai commis dans les nombres une légère erreur, il n'en resterait pas moins constant, et c'est là tout ce qu'il m'importait de considérer, que l'on pouvait éloigner entre d'assez grandes limites le corps lumineux du verre pour cesser d'avoir une image nettement terminée. Telle est, Messieurs, la réponse à l'objection qui m'est faite. Ce n'est pas la partie faible du paragraphe $3^{\mathrm{e}}$ de mon mémoire que ces Messieurs ont attaquée. Il y avait d'autres objections beaucoup plus fondées à me faire, et je me les suis faites très peu après l'envoi de mon mémoire. Aussi me repentais-je beaucoup alors de vous l'avoir envoyé si promptement et je recommençais dès lors mon travail. Je veux parler ici du solide formé par l'image et dont je croyais que la [mot illisible] ferait différents facteurs selon la distance. Et bien, ce solide là 
n'existe pas ou du moins seulement avec des dimensions relatives différentes de celles de l'objet, vu que son épaisseur ou pour mieux dire son étendue antéro-postérieure est toujours extrêmement raccourcie; au lieu que la hauteur et la largeur doivent conserver respectivement les mêmes grandeurs relatives que dans l'objet. La cause de mon erreur a donc été de croire que la diminution de grandeur dans l'image était pour toutes les dimensions proportionnelle à l'augmentation de la distance de l'objet. Cette objection que je me fis à moi-même fut bientôt confirmée par des expériences que je fis à cet effet, mais que je me dispenserai de rapporter vu que la chose est évidente par elle-même. Comme je vous l'ai dit, très chers collègues, j'ai repris le problème en entier, et je ne désespére pas d'arriver à sa solution. Sans m'en tenir à de simples aperçus théoriques et explications plus ou moins approchées, j'ai voulu chercher à appliquer les mathématiques pour parvenir à la solution. J'ai eu en conséquence de nombreuses expériences à faire pour déterminer les données du problème: déterminer la courbure et la force réfringente de la cornée, de l'humeur aqueuse, du cristallin, du corps vitré, la forme de tout l'œil lui-même. Le cristallin seul m'a donné une peine infinie et je n'ai pas encore atteint un point où je veux parvenir après avoir déterminé la forme et le pouvoir réfringent de chacune des couches qui composent cette admirable lentille. Il faudra par des intégrations déterminer le foyer de la totalité de ce corps, puis comparer le résultat du calcul avec celui de l'expérience. Voilà, Messieurs, une idée du probléme dont je poursuis la solution. Vous pouvez juger de ses difficultés; aussi, seul avec les faibles moyens dont je pouvais disposer, m'eut-il été impossible d'y arriver, sans la bonté vraiment extraordinaire (et dont je conserverai toujours la plus vive reconnaissance) avec laquelle le célèbre Monsieur Biot m'a honoré de ses précieux conseils, et m'a fourni tous les moyens nécessaires de parvenir à mon but. En effet, il a mis à ma disposition tout le cabinet de physique de la Faculté des Sciences, avec les excellents instruments d'optique construits par Monsieur Cauchoix; et en partant pour l'Ecosse (où vous savez sans doute qu'il a été envoyé pour mesurer la longueur du pendule à secondes), il a pris les instruments nécessaires pour déterminer les pouvoirs réfringents des yeux de poissons et d'oiseaux de mer qu'il aura en abondance dans ces régions, données qu'il me donnera pour insérer dans mon mémoire. Il ne sera de retour qu'en novembre. Nous avons composé avec $\mathrm{Mr}$ Macaire un mémoire sur l'action des purgatifs et qui est prêt à vous envoyer, quand il se présentera une occasion. Mais quant à mon second mémoire, vous voyez que probablement je ne pourrai vous l'envoyer avant la fin de notre seconde année: en conséquence si la Sociéte ne me donne pas une dispense je me verrai obligé de partager en deux le mémoire que je comptais lui envoyer. J'y travaille sans relâche depuis plusieurs mois mais, comme vous le savez, à l'impossible nul n'est tenu. C'est par là que je terminerai cette lettre. Je vous prie de recevoir, très chers Collègues, l'assurance des sentiments d'amitié avec lesquels j'ai l'honneur d'être, Votre dévoué compère, Charl. Chossat.»

III. Lettre à Monsieur Fred. Soret, ex-président de la Société des Amateurs des Sciences Naturelles à Genève. «Paris, le 2 mars 1819, Très chers collègues, Etant il y a quelques mois à Montauban je vous fis savoir que j'avais rédigé un Mémoire prêt à vous être envoyé, sur la courbure de la cornée chez le Boeuf; j'ai l'honneur de vous annoncer aujourd'hui que j'ai fait un nouveau mémoire sur l'œil de bœuf, que j'ai rédigé à Montauban comme le premier et que je vous enverrais si j'en avais l'occasion. Ces deux mémoires réunis en un seul ont été lus par moi à la Société philomatique dans sa séance du 21 novembre 1818; trois commissaires, Messieurs Bu... [illisible] et Edwards ont été nommés pour en faire leur rapport à la Société, et j'ai eu la 
satisfaction de voir qu'ils avaient jugé mon travail assez favorablement pour que sur leur proposition, la Société philomatique m'ait accordé le titre de son correspondant. Je serais infiniment flatté que Monsieur le Président daignat m'honorer d'une lettre dans laquelle il me ferait connaître quel est l'état actuel de notre Société: il y a près de deux ans que je suis sans nouvelle à cet égard et vous conviendrez, très chers collègues, que cela est bien long pour quelqu'un qui prend autant d'intérêt que moi à notre société. Monsieur de la Rive à son passage à Paris m'a assuré que la Société helvétique nous avait reçu dans son sein; ce serait un évènement infiniment honorable pour nous, et trop remarquable dans l'histoire de notre société pour que vous ne m'en eussiez point instruit comme tous les autres membres absents. J'attends sur cela comme sur tout le reste des éclaircissements que Monsieur le Président voudra bien me faire parvenir. Recevez, mes très chers collègues, l'assurance de mon parfait attachement. Ch. Chossat. Je reçois à l'instant même d'excellentes nouvelles de notre cher collègue M. Coindet.»

IV. Lettre au Dr L.A. Gosse ${ }^{35}$ à Genève. «Londres, 11 Gr Marlborough Str., 18 Janv. 1821. Monsieur et cher Ami, Je crains en vérité d'avoir passé dans votre esprit pour un homme bien impoli, pour avoir tardé si longtemps à répondre à votre lettre du mois d'août dernier, et à vous témoigner mes remerciements des deux lettres de recommandation qu'elle contenait. Mais j'espère que vous m'exuserez en apprenant que par je ne sais trop quelle raison ces deux lettres sont restées à Paris, et que ce n'est qu'à la suite d'un avis que je reçus ici de l'administration des Postes que j'ai pu les faire retirer. J'ai reçu beacoup d'accueil de la part de Mess ${ }^{\text {rs }}$ Brodie et Gordon: ils se sont beaucoup informé de vous et m'ont chargé de les rappeler à votre souvenir. Vous avez si bien vu ce pays, qu'il serait difficile de vous en dire quelque chose qui put vous intéresser par sa nouveauté en ce qui regarde notre profession au moins: c'est pourquoi je renonce à vous entretenir des établissements sanitaires; je préfère vous parler d'un médicament introduit récemment dans la pratique et qui n'est peut-être pas encore parvenu à votre connaissance à cause du peu de temps dont il en est question ici (environ 6 semaines); je veux parler de l'huile de croton. L'Huile de croton est une huile exprimée du Croton Tiglium qui croît dans les Indes Orientales; elle a l'odeur, la couleur et la consistance de l'huile de ricin, en sorte qu'il est à craindre qu'on ne se serve de cette dernière pour la falsifier. Sa saveur cependant suffit pour la faire distinguer de l'Huile de ricin car une ou deux gouttes mises sur la langue y laissent une saveur extrêmement âcre, comme brûlante, et détermine un flux de salive assez prolongé. On se sert beaucoup de cette huile dans les Indes Orientales: Mr Conwell dit qu'il l'y a employée pendant 18 mois: le 23 juin 1820, il en expédia de Madras une certaine quantité à Mr Arthur Short, apothecary \& chemist, 11 Ratcliff Highway, avec ordre de la distribuer dans les hôpitaux de Londres pour en faire des expériences comme purgatif. Il y a joint une courte instruction sur la manière de l'administrer, instruction que j'ai eu quelques instants entre les mains et dont j'ai extrait ce qui suit. Pour une personne adulte, la dose ordinaire est de 1 goutte: mais quelque fois on pousse jusqu'à 2 ou 3 gouttes. Ce médicament opère dans une demi-heure environ et produit dans les fièvres bilieuses une énorme quantité (amazing quantity) de matières. Après l'avoir employé pendant 18 mois, il le recommande as the speediest, most effectual \& safe purge he ever employed. Sur la même instruction, dans un paragraph signé Dr White, il est dit qu'une trop forte dose produit le vomissement; et que quelques-uns corrigent ce médicament par le cachou. Enfin dans un article signé Marshal surgeon, imprimé sur la même feuille on y dit que 1 goutte de cette huile divisée en deux pilules 
agit avec la même efficacité que $3 \mathrm{~g}$ de Jalop ou [illisible] de Calomel ou enfin $3 \mathrm{~g}$ de sel d'Epsom; qu'une demi-heure après avoir pris cette dose, le malade ressent quelque mouvement dans le canal intestinal, et qu'une demi-heure plus tard il rend une selle, que les évacuations alvines sont invariablement liquides et copieuses; que dans 1/10 des cas environ il a observé des [mot illisible] et dans $1 / 30$ des nausées. Il ajoute que la petitesse de la dose et la rapidité de l'action doivent rendre ce médicament fort avantageux dans l'apoplexie, et que dans un cas de coma, il lui a vu déterminer plusieurs selles liquides. Mr. Martindale (que vous connaissez probablement), apothicaire au London Hospital, m'a montré cette huile de croton. Désirant en faire l'essai sur lui-même, il en prit à minuit deux gouttes qui lui laissèrent une saveur très âcre et causèrent beaucoup de salivation: à 4 heures du matin il se réveilla avec beaucoup de malaise, vomit et eut une légère selle: à 6 heures il se réveilla de nouveau et eut une seconde selle, mais beaucoup plus copieuse que la première: je le vis à 11 heures du matin et alors il éprouvait encore beaucoup de dégoût et de malaise, et paraissait fort irascible. Il paraît que la simple précaution de prendre la goutte d'huile sur un morceau de sucre suffit pour qu'on ne s'aperçoive pas de sa saveur: c'est au moins ce qui a paru à un pharmacien qui en prit en ma présence. Enfin pour terminer cette petite note, Mr Wheeler de St Bartolomew Hospital, m'a dit que Geoffroy parlait de cette huile de croton et disait qu'une goutte était une dose suffisante, sur quoi Lewis observe que Geoffroy s'était probablement trompé et que ce devrait être $3 \mathrm{~g}$ qui était la véritable dose, ce qui montre que Lewis n'en a jamais vu administrer. Voilà, cher collègue, ce que j'ai pu apprendre, touchant à l'huile de croton: vous pourrez en faire part à nos amis et si vous le jugez convenable à Messieurs de la Société de Médecine, s'ils n'ont pas encore reçu de communication à cet égard et si vous croyez que cela puisse les intéresser. Veuillez présenter mes amitiés au Dr Coindet, à mon ami le Dr Prevost et à Macaire, et agréez l'expression des sentiments d'estime et d'amitié avec lesquels j'ai l'honneur d'être Votre dévoué, Ch. Chossat. On trouve quelques détails sur le Croton Tiglium dans l'Hortus malaboricum et l'Hortus amboinensis».

V. Lettre au président de la Société médicale de Genève. «Genève, le 12 janvier 1830. Monsieur le Président, Comme je veux point m'assujettir à la Présence réelle (pour me servir d'une expression qui vient d'être consacrée par le rapport annuel de la Société), non plus qu'à la présentation de Mémoires à des époques fixes et régulières, je vous prie de vouloir faire agréer à la Société Médicale du Canton ma démission de Membre de cette société. Veuillez cependant lui exprimer mes regrets de ce que les règlements ne m'autorisent pas à lui demander de transformer mon titre d'associé en celui de correspondant, ou en tel autre qui me permette de conserver avec elle des relations que j'aurais aimé à ne pas voir interrompues entièrement. Agréez, Monsieur le Président, l'assurance de l'amitié et de la parfaite considération de Votre trés humble serviteur, Ch. Chossat, M.D.» 


\section{Notes}

1 Pour l'histoire de la physiologie à la Faculté de Médecine, voir Centenaire de la Faculté de Médecine de l'Université de Genève (1876-1976). Documents rassemblés par Marc Cramer et Jean Starobinski, bio-bibliographies par Marc-A. Barblan. Editions Médecine et Hygiène, Genève 1978, p. 37-57.

2 K. Kilgus: Charles Chossat. 1796-1875. Erkenntnisse eines Genfer Experimentalphysiologen in der ersten Hälfte des 19. Jahrhunderts. Juris Druck \& Verlag, Zürich 1967. 35 p.

3 Volume 9, 1975, p. 140-154.

4 Je remercie le conservateur des manuscrits de la Bibliothèque publique et universitaire (pour les lettres I-IV) et la directrice du Musée d'histoire des Sciences (pour la lettre V et la photo de Chossat) pour l'autorisation qui'ils nous ont donnée de reproduire ces documents. La B.P.U. possède également en manuscrit un mémoire sur le pouvoir réfringent des milieux de l'œil, qui correspond de près au texte de l'article 1 de l'annexe 1.

5 Jean-François Coindet (1774-1834) pratiqua la médecine à Genève après des études entreprises à Edimbourg. Il est surtout connu pour avoir introduit en 1820 l'emploi de l'iode dans le traitement du goitre. Voir J. Olivier: Les origines de la Sociéte médicale de Genève et le $\mathrm{D}^{\mathrm{r}}$ Jean-François Coindet. Revue médicale de la Suisse romande 68, 1948, p. 326-334.

6 Ne pas confondre avec son frère Henri (1800-1885), auteur des Leçons sur la physiologie et l'anatomie comparées de l'homme et des animaux. D'abord professeur d'histoire naturelle à Bruges, William Edwards (1772-1842) termina ses études médicales à Paris, puis entreprit des recherches physiologiques. Parmi ses travaux, signalons Sur la structure de l'œil (1814) et Influence que la température exerce sur l'économie, sur l'influence vivifiante de l'air et sur la transpiration (1817). Noter la similitude de ces thèmes avec ceux auxquels s'attelle le jeune Chossat. William Edwards se consacra par la suite à l'ethnologie, l'anthropologie et la linguistique.

7 E. H. Ackerknecht: La médecine à Genève surtout dans la première moitié du XIX ${ }^{\mathrm{e}}$ siècle. Comptes rendus du XIX ${ }^{e}$ Congrès international d'Histoire de la Médecine. Karger, Bâle 1966, p.420-425. E. Thomas. La médecine à Genève au début du XIX siècle. CIBA, Bâle $1937,40 \mathrm{p}$.

8 Voir lettre II.

9 Pour s'occuper de son père dont l'état de santé laissait à désirer.

10 Sur le rôle de l'Académie des sciences et du prix Montyon décerné par elle dans l'émergence de la physiologie expérimentale en France, voir P. Elliott, Vivisection and the Emergence of Experimental Physiology in Nineteenth-century France. In: Vivisection in Historical Perspective, edited by N.A. Rupke, Croom Helm, London 1987, p.48-77. Sur les lauréats voir N. Egli, Der «Prix Montyon de Physiologie expérimentale» im 19. Jahrhundert. Juris Druck \& Verlag, Zürich 1970, 90 p. Magendie fit pendant de très nombreuses années partie de la commission d'attribution du prix Montyon. Parmi les premiers lauréats, signalons William Edwards (1820, 1821), ainsi que Prevost et Dumas (1824). Le prix de 1825, qui s'élevait à 895 francs, faillit être partagé entre Chossat et Flourens, déjà lauréat en 1823 et 1824. Magendie fit pencher la balance en faveur du seul Chossat, arguant du fait que le travail de Flourens, dont il reconnaissait par ailleurs la qualité, ne représentait qu'une poursuite de ses recherches antérieures et qu'il avait, par conséquent, déjà été récompensé. 
11 Les expériences n'étaient pas terminées en 1831, car, écrit Chossat, quand j'eus fait à peu près la moitié de mes observations [sur l'oscillation diurne], je lus, en 1831 je crois, une note à ce sujet à la Société de physique et d'histoire naturelle de Genève; mais je ne l'ai point fait imprimer dans la collection de ses Mémoires (Recherches, note p. 533). Elles étaient certainement achevées en 1835 puisque, renonçant à faire figurer dans les recherches ses études sur le régime sans sucre, qui paraîtront sous forme résumée en 1843, Chossat précise: les expériences que j'aurais eu à rapporter sur ce sujet comme, au reste, toutes celles de ce Mémoire, sont déjà complètement terminées depuis six à sept ans (Recherches, note p. 495-496).

12 Sur le rôle et l'importance des questions relatives à la bioénergétique dans la physiologie du $\mathrm{XIX}^{\mathrm{e}}$ siècle, voir, par exemple: G. Canguilhem: La constitution de la physiologie comme science. In: Etudes d'histoire et de philosophie des sciences, J.Vrin, Paris 1968, p. 226-273; W. Coleman: Biology in the Ninteenth Century, Problems of Form, Function and Transformation, Cambridge University Press, 1977, p.118-159. Sur le problème particulier du siège de la chaleur animale, voir E. Mendelsohn. The controversy over the site of heat production in the body. Proceedings of the American Philosophical Society 105, 1961, p.412-420.

13 Le Mémoire est signé Ch.Chossat, M.-D. à Pise, en Toscane. Chossat y écrit: Presque toujours en voyage depuis quatre ans, les expériences de ce mémoire ont été exécutées pendant les résidences que je faisais dans les différents lieux où je me trouvais momentanément fixé; cela m'a privé de plusieurs secours qui m'auraient été bien nécessaires (note p. 186).

14 L'inanitiation, c'est l'examen des faits qui se rapportent à la production graduelle de l'inanition. En ce sens l'inanition, à proprement parler, n'est que la fin de l'inanitiation (Recherches, p. 446).

15 La chaleur se prenait avec un thermomètre centigrade à boule, enfoncé de 1-2 $\mathrm{cm}$ dans le cloaque de l'animal et laissé en place pendant cinq minutes.

16 Recherches, p. 554.

17 Recherches, p. 505.

18 Après avoir pesé chaque organe, Chossat pratiquait une dessication à l'étuve, puis refaisait toutes les pesées, pour obtenir les poids secs.

19 Chossat précise qu'il a exécuté toutes ses expériences seul et sans aucune assistance (Recherches, p. 599). Rappelons aussi qu'une partie du travail a été entreprise alors que Chossat voyageait. L'expérience avec une tourterelle, qui dura du 4 avril au 30 juin 1828 est illustrative à cet égard. Elle débuta à Naples, puis se déroula à Rome, Viterbe, Florence, Pise, pour se terminer dans le grand-duché de Bade (Recherches, p. 633-636).

20 Recherches, p. 632.

21 Cette lettre est reproduite dans Charles Borgeaud, L'Académie et l'Université au XIX ${ }^{e}$ siècle. 1814-1900. (Histoire de l'Université de Genève, tome 3). Georg et Cie, Genève 1934, note p. 352.

22 La lettre à James Fazy dans laquelle Mayor père s'explique ne manque pas d'humour: $I l$ m'est pénible d'être obligé de refuser le professorat auquel j'ai longtemps rêvé, mais c'est trop tard; tout ce que je puis faire à présent c'est de lui livrer mon corps pour servir à deux ou trois leçons; mais soyez sûr que je le ferai le plus tard possible (Ch. Borgeaud, op.cit., note p. 362). On attribue habituellement à Mayor père d'avoir le premier entendu et décrit en 1818 les bruits du cœur fœtal, signe qui permet de distinguer si un fœetus est vivant ou mort. Mayor 
fils, devenu professeur aimait, parait-il, raconter à ses étudiants que son cœur était le premier dont les battements furent entendus avant la naissance.

23 Pour Mayor fils, voir la nécrologie parue dans le Revue Médicale de la Suisse Romande 19, 1899 , p. 364-366.

24 Jean Charles Coindet (1796-1876), fils de Jean-François (note 5). Docteur en médecine d'Edimbourg, revenu à Genève en 1823, il s'occupa surtout d'aliénés à l'asile des Vernaies, dont il fut longtemps médecin en chef. Destitué par le Conseil d'Etat en 1856, il fut l'un des fondateurs de La Métairie, laquelle demeure encore en activité. Avec son père J.Ch.C. fut parmi les fondateurs de la Société médicale, en 1823. La Société de physique et d'histoire naturelle date, elle, de 1790.

25 Bulletin de la Société médicale de la Suisse Romande 9, 1875, p.151.

26 Léon Revilliod (1835-1918), docteur en médecine de Paris (1865), fut médecin en chef de l'Hôpital cantonal (1870-74), puis dès la création de la Faculté, nommé professeur de clinique médicale (1876-1899).

27 Compte-rendu des travaux de la Société médicale du canton de Genève pendant l'année 1875, in Bulletin de la Société médicale de la Suisse Romande 10, 1876, p.9.

28 Librairie Baillière et fils, Paris 1876, p.205. Bernard cite Brodie et Chossat, tout en critiquant leur interprétation attribuant exclusivement la calorification à une influence du système nerveux.

29 Félix Alcan, Paris, 3, 1898, p. 86.

30 Prevost fut d'abord professeur de thérapeutique à la Faculté de Médecine (1876-1896), puis, à la mort de Schiff, devint professeur de physiologie (1897-1913). Une récente biographie est due à R. Mayor. Jean-Louis Prevost «junior». Revue du Vieux Genève, $\mathrm{XI}^{\mathrm{e}}$ année - No 11,1981 , p.11-18.

31 C. Montandon: Science et société à Genève aux XVIII ${ }^{\mathrm{e}}$ et $\mathrm{XIX}^{\mathrm{e}}$ siècles. Gesnerus 32, fasc. $1-2,1975$, p. 16-34.

32 Prevost fut secrétaire général du $1^{\text {er }}$ Congrès des sciences médicales (Genève, 1877) et président du Congrès des neurologistes de langue française (Genève, 1907). De 1904 à 1913, il siégea dans le Comité de l'Union internationale des sciences physiologistes. Il fut nommé docteur honoris causa des universités de Lausanne et de Genève.

33 Pierre Prevost (1751-1839). Professeur de philosophie à Berlin, puis de belles-lettres, de philosophie et de physique générale à l'Académie de Genève. Ses travaux les plus importants concernent le rayonnement thermique, la magnétisme et le système solaire.

34 Créée en 1815, cette Société regroupait une dizaine de jeunes savants en herbe. Présidée par Frédéric Soret (1795-1865), naturaliste et numismate, elle se fondit par la suite dans la Société helvétique des sciences naturelles.

35 André Louis Gosse (1791-1873). Docteur en médecine (Paris 1816), il fut avec J.L. Prevost et J.-P. Dupin un des fondateurs du Dispensaire des médecins (1820) et assura avec J.-F. Coindet et F.-I. Mayor père, la chronique scientifique du Journal de Genève. Son père Henri Albert (1753-1816) fut en 1815 un des fondateurs et le premier président de la Société helvétique des sciences naturelles. 


\section{Summary}

During his formative years - he was a contemporary of J.-L.Prevost and studied medicine in Paris - Charles Chossat (1796-1875) came under the spell of F.Magendie and W. Edwards. For the next two decades, including during the first years following his return to Geneva, he performed quantiative physiological experiments on body temperature, on urine output and on food deprivation. On two occasions, in 1825 and in 1843, he was given the Montyon prize of experimental physiology awarded by the Paris Academy of Sciences. Although he was in good terms with the radical authorities which came to power in Geneva in 1846, Chossat was not given a position to pursue his physiological studies. A different sort of physiology-surgical in inspiration and not prone to animal experimentation - was dominant in Geneva during the period when F.-I. Mayor taught at the Faculty of Sciences of the Academy (1849-1875). It was only after 1876 - when Schiff moved from Florence to Geneva - that experimental physiology would develop at the newly created Geneva Faculty of Medicine.

Prof. J. J. Dreifuss

Département de Physiologie Centre Médical Universitaire 9, avenue de Champel

CH-1211 Genève 4 